\title{
Human Krüppel-like factor 11 inhibits human proinsulin promoter activity in pancreatic beta cells
}

\author{
X. Niu • N. Perakakis $\cdot$ K. Laubner $\cdot$ C. Limbert $\cdot$ \\ T. Stahl • M. D. Brendel • R. G. Bretzel • J. Seufert • \\ G. Päth
}

Received: 7 November 2006 / Accepted: 26 February 2007 / Published online: 4 May 2007

(C) Springer-Verlag 2007

\begin{abstract}
Aims/hypothesis The Krüppel-like factor 11 (KLF11; TIEG2), a pancreas-enriched Sp1-like transcription factor, is a known negative regulator of pancreatic exocrine cell growth. A recent study indicated KLF11-induced activation of the human proinsulin promoter (hInsP).

Materials and methods We investigated the functional role of KLF11 in pancreatic beta cells.

Results Endogenous KLF11 mRNA expression was found in whole rat pancreas, human pancreatic islets and INS-1E beta cells and was profoundly reduced by high glucose in INS-1E. Cotransfections of INS-1E and beta-TC3 beta cells with a human (h)KLF11 expression plasmid and an hInsP-driven reporter plasmid resulted in a substantial dose-dependent and
\end{abstract}

This work is dedicated to X. Niu who died in December 2006 in China under tragic circumstances. An excellent research talent has died and left us in deep mourning.

X. Niu $\cdot$ N. Perakakis $\cdot$ K. Laubner $\cdot$ C. Limbert $\cdot$ T. Stahl $\cdot$

J. Seufert $\cdot$ G. Päth $(\bowtie)$

Laboratory B9, Division of Endocrinology and Diabetology,

Department of Internal Medicine II,

University Hospital of Freiburg,

79106 Freiburg, Germany

e-mail: guenter.paeth@uniklinik-freiburg.de

X. Niu

Department of Hepatobiliary Surgery,

First Hospital of Xi' an Jiaotong University,

Xi'an, People's Republic of China

M. D. Brendel • R. G. Bretzel

Islet Transplantation Centre, Department of Internal Medicine III,

University of Giessen,

Giessen, Germany glucose-independent inhibition of proinsulin promoter activity. 5'-deletion of hInsP demonstrated that hKLF11 acts via DNA sequences upstream of -173 and requires the beta cellspecific transcription machinery, since hKLF11-mediated inhibition of promoter activity was abolished in HEK293 cells. Besides a previously described GC box, we further identified a CACCC box within the hInsP, both putative KLF11-binding motifs. Electrophoretic mobility shift analysis (EMSA) verified binding of in vitro translated hKLF11 to the GC box, but neither hKLF11-induced inhibition nor basal hInsP activity was altered by mutation or $5^{\prime}$-deletion of the GC box. In contrast, CACCC box mutation substantially reduced basal promoter activity and partially diminished hKLF11 inhibition, although binding of in vitro translated hKLF11 to the CACCC box could not be verified by EMSA. Conclusions/interpretation In rodent beta cell lines, we demonstrate $h K L F 11$-overexpression of human proinsulin gene expression and characterise a prominent role for the CACCC box in maintaining basal proinsulin promoter activity.

Keywords Beta-TC3 $\cdot$ CACCC box $\cdot$ GC box $\cdot$ Human proinsulin promoter $\cdot$ HEK293 $\cdot$ INS-1 $\cdot$ KLF11

$\begin{array}{ll}\text { Abbreviations } \\ \text { EMSA } & \text { electrophoretic mobility shift analysis } \\ \text { hInsP } & \text { human proinsulin promoter } \\ \text { HPRT } & \text { hypoxanthine phosphoribosyltransferase } \\ \text { KLF } & \text { Krüppel-like factor } \\ \text { MAOB } & \text { monoamine oxidase B } \\ \text { PDX-1 } & \text { pancreatic duodenal homeobox protein-1 } \\ \text { qPCR } & \text { quantitative real-time PCR } \\ \text { SEAP } & \text { secreted alkaline phosphatase } \\ \text { TIEG } & \text { TGF- } \beta \text {-inducible early response gene }\end{array}$




\section{Introduction}

Krüppel-like transcription factor (KLF) 11 is a member of the Sp1-like transcription factor family, which is defined by the presence of three conserved DNA-binding C-terminal zinc finger domains and variant $\mathrm{N}$-terminal domains [1-3]. $\mathrm{KLF} / \mathrm{Sp}$ 1-like proteins bind with different selectivity to GC box or CACCC box promoter elements and take part as activators or repressors in virtually all aspects of cellular function, including cell proliferation, apoptosis, differentiation and neoplastic transformation.

Within the KLF/Sp1-like family KLF9, KLF10, KLF11, KLF13 and KLF16 are characterised by the existence of a repressor domain which interacts with the scaffold corepressor mammalian (m)Sin3A (SID or SID/R1 for KLF10 and KLF11) as a common structural feature [4]. SID/R1 mediates chromatin modification and other repressor mechanisms via recruitment of histone deacetylases and the nuclear receptor corepressor N-CoR, respectively [2]. Among the above-mentioned KLF proteins, KLF10 and KLF11 are further characterised by the existence of two additional repressor domains R2 and R3 [5] and TGF- $\beta$-induced expression $[6,7]$. For this reason KLF10 and KLF11 form the TGF- $\beta$-inducible early response gene (TIEG) subfamily and were alternatively named TIEG1 and TIEG2, respectively. Due to parallel cloning, KLF11 was also named FKLF [8].

In the adult organism, KLF11 is ubiquitously produced, but enriched in muscle and pancreas [6]. Within the pancreas KLF11 has been described as a negative regulator of exocrine cell proliferation in transgenic mice with acinar cell-specific KLF11 overproduction in vivo and PANC1 epithelial cancer cells overexpressing KLF11 in vitro [9]. As a result, KLF11 transgenic mice develop a significantly smaller exocrine pancreas than controls due to reduced proliferation and enhanced apoptosis. Albeit a smaller exocrine pancreas, only mild changes in tissue architecture could be observed and levels of the acinar enzymes remained normal. In vivo and in vitro reduced proliferation is accompanied by enhanced apoptosis. This is, at least in part, explainable by KLF11-mediated downregulation of the scavengers superoxide dismutase 2 and catalase 1, resulting in increased susceptibility of cells to oxidative stress.

The role of KLF11 within the endocrine pancreas remained unestablished until recently Neve et al. [10] reported that, in beta cell lines, high glucose conditions stimulate KLF11 mRNA expression and cotransfected human (h)KLF11 can activate the human proinsulin promoter (hInsP). In our own experiments, however, endogenous KLF11 mRNA levels were reduced by high glucose in INS-1E beta cells. Furthermore, cotransfected hKLF11 dose-dependently and glucose-independently inhibits the activity of hInsP in INS-1E and beta-TC3 beta cells. Sequence analysis of hInsP not only retrieved the previously described GC box but also identified a CACCC box element, both putative binding sites of KLF11. Since our results are in contrast to those of Neve et al. [10] and to better understand how hKLF11 regulates human insulin gene expression, we here further studied the functional role of the GC and CACCC box by electrophoretic mobility shift analysis (EMSA), mutation and 5'-deletion constructs of the hInsP in INS-1E and beta-TC3 beta cells.

\section{Materials and methods}

Cell culture Cell lines were routinely cultured as follows. Rat INS-1E: RPMI 1640 containing 10\% heat-inactivated fetal bovine serum (FBS), $1 \mathrm{mmol} / \mathrm{l}$ sodium pyruvate, $2 \mathrm{mmol} / \mathrm{l}$ L-glutamine, $100 \mathrm{U} / \mathrm{ml}$ penicillin, $100 \mu \mathrm{g} / \mathrm{ml}$ streptomycin and $50 \mu \mathrm{mol} / 1 \quad \beta$-mercaptoethanol. Media contained $11.1 \mathrm{mmol} / 1$ glucose unless otherwise stated. Mouse betaTC3 beta cells and human HEK293 cells: DMEM containing 10\% FBS, $25 \mathrm{mmol} / 1$ glucose, $2 \mathrm{mmol} / 1 \mathrm{~L}$-glutamine, $100 \mathrm{U} / \mathrm{ml}$ penicillin and $100 \mu \mathrm{g} / \mathrm{ml}$ streptomycin. INS-1E beta cells were kindly provided by C. B. Wollheim (University Medical Center, Geneva, Switzerland).

RT-PCR detection of KLF11 mRNA expression in pancreatic tissues and cells Total RNA extraction was performed using Trizol (Invitrogen, Karlsruhe, Germany) for tissue or RNeasy Mini Kit (Qiagen, Hilden, Germany) for cells. RNA samples were reverse transcribed into cDNA in the presence (RT+) or absence (RT-) of Superscript II Plus using oligo-(dT) primers (both Invitrogen). RT- samples served as controls for the absence of genomic DNA contamination. Primers: rat (r)KLF11, forward 5'-GAAGC GGCACGACAGCGAAAG-3' and reverse 5'-AGCTC TGGGCTCTGAGGAGGAGTT-3' [annealing temperature (Ta), $64^{\circ} \mathrm{C}$; product length, $250 \mathrm{bp}$ ]; hKLF11, forward 5'GGTGACCTGTTGCGGATAAG-3' and reverse 5'-CACA GGGATCATCTGGCAAAGGA- $3^{\prime}\left(\mathrm{Ta}, 60^{\circ} \mathrm{C}\right.$; product length, $687 \mathrm{bp}$ ). PCR conditions: $94^{\circ} \mathrm{C}$ for $2 \mathrm{~min}$; 35 cycles of $94^{\circ} \mathrm{C}$ for $30 \mathrm{~s}$, primer-specific Ta for $30 \mathrm{~s}$ and $72^{\circ} \mathrm{C}$ for $30 \mathrm{~s}$; $72^{\circ} \mathrm{C}$ for $2 \mathrm{~min}$.

Plasmid construction The hKLF11 coding sequence was amplified from cDNA derived from human islets of Langerhans by using high fidelity Pwo Master (Roche, Mannheim, Germany). Primers: forward 5'-CACGATGCAC ACGCCGGACTTC-3' and reverse 5'-GCTAGCAAAAT CCCATGAGTGATGTCCTAATGG- $3^{\prime}$. PCR conditions: $94^{\circ} \mathrm{C}$ for $2 \mathrm{~min}$; 30 cycles, $94^{\circ} \mathrm{C}$ for $30 \mathrm{~s}-54^{\circ} \mathrm{C}$ for $30 \mathrm{~s}-72^{\circ} \mathrm{C}$ for $3 \mathrm{~min}$. The resulting $h K L F 11$ coding sequence was subcloned into pcR2.1-TOPO by using a TOPO TA Cloning kit (both Invitrogen) and subcloned into the CMV promoter-driven pcDNA3.1+ (Invitrogen) to obtain the hKLF11-pcDNA3.1+ 
expression plasmid. The -881 to +54 hInsP fragment was amplified with a BD Advantage HF PCR Kit (BD Biosciences Clontech, Heidelberg, Germany) from human genomic DNA which was extracted from whole blood using a QIAamp DNA Blood Mini Kit (Qiagen). Primers: forward 5'-TCCCTCACTCCCACTCTCCCAC-3' and reverse 5'TTCGAATTGGAACAGACCTGCTTGATGGCC-3'. PCR conditions: $94^{\circ} \mathrm{C}$ for $3 \mathrm{~min} ; 28$ cycles of $94^{\circ} \mathrm{C}$ for $30 \mathrm{~s}$, $55^{\circ} \mathrm{C}$ for $30 \mathrm{~s}$ and $68^{\circ} \mathrm{C}$ for $90 \mathrm{~s} ; 68^{\circ} \mathrm{C}$ for $2 \mathrm{~min}$. The resulting $-881+54 \mathrm{hInsP}$ fragment was subcloned into pcR2.1-TOPO plasmid using a TOPO TA Cloning kit (both Invitrogen) and subcloned into pSEAP2-Basic (BD Biosciences Clontech) to obtain the $-881 \mathrm{hInsP-pSEAP}$ reporter plasmid (SEAP, secreted alkaline phosphatase). $-881 \mathrm{hInsP}-$ pSEAP was used for generation of 5 -deleted hInsP fragments $(-387,-355,-323,-254,-173,-101$ and -85$)$ with an identical $3^{\prime}$-end $(+54)$ by PCR, which were then subcloned via pcR2.1-TOPO into pSEAP2-Basic. GC box and CACCC box mutations were created using a QuickChange Site-Directed Mutagenesis Kit (Stratagene, Heidelberg, Germany). The GC box was mutated from 5'-CCC GCCCT-3' into 5'-CCCGAACT-3' and the CACCC box from 5'-CCCACCCC-3' into 5'-CCCGATTCC-3'. Accuracy of all sequences and mutations was checked by sequencing.

Protein extraction and in vitro translation For generation of whole cell extracts, freshly prepared RIPA buffer (Upstate/ Biomol, Hamburg, Germany) containing one tablet per $10 \mathrm{ml}$ Complete Mini EDTA-free protease inhibitor cocktail (Roche) was used. Nuclear extracts were acquired according to Schreiber et al. [11]. hKLF11 was in vitro translated from hKLF11-pcDNA3.1+ using a TNT Quick Coupled Transcription/Translation System (Promega, Mannheim, Germany). Control reactions were performed in the absence of hKLF11-pcDNA3.1+.

Western blot analysis Ten micrograms of protein from whole cell extract and nuclear extract or $1 \mu l$ in vitro translated reactions were separated on 10\% PAGE-SDS gels. Proteins were transferred to polyvinylidene difluoride membranes by semi-dry blotting using Towbin transfer buffer. KLF11 was detected using a goat polyclonal antiserum (TIEG2 C-12, sc-23162; Santa Cruz, Heidelberg, Germany). Visualisation was achieved by a horseradish peroxidase-linked rabbit anti-goat secondary antibody (DAKO, Hamburg, Germany) and ECL Western Blotting Detection Reagent (Amersham, Freiburg, Germany).

SEAP reporter gene experiments INS-1E, beta-TC3 and HEK293 cells were seeded in six-well plates at a density of $3 \times 10^{5}$ cells per well. Next day, cells were transiently transfected using Metafectene (Biontex, Martinsried/Planegg,
Germany). The proportion of DNA ( $\mu \mathrm{g})$ to Metafectene $(\mu l)$ was 1:2. Each plasmid was transfected at a concentration of $0.5 \mu \mathrm{g}$ unless otherwise stated. After $48 \mathrm{~h}$, supernatants were collected for measurement of SEAP using a BD Great EscAPe SEAP Chemiluminescence Detection Kit (BD Biosciences Clontech).

Quantification of insulin gene expression in INS-1E beta cells in response to KLF11 overexpression INS-1E cells were seeded in six-well plates at a density of $3 \times 10^{5}$ cells per well in the presence of $11.1 \mathrm{mmol} / 1$ glucose. Next day, cells were transiently transfected with either rKLF11pcDNA3.1+ or pcDNA3.1+ (mock control level) using Metafectene Pro (Biontex). The proportion of DNA $(\mu \mathrm{g})$ to Metafectene Pro $(\mu \mathrm{l})$ was 1:2. Each plasmid was transfected at a concentration of $1 \mu \mathrm{g} / \mathrm{well}$. After $48 \mathrm{~h}$, cells were harvested for analysis of monoamine oxidase B gene $(M A O B)$, insulin-1 (Ins1) gene and insulin-2 (Ins2) gene mRNA expression levels using quantitative real-time PCR (qPCR) (see below).

Quantification of KLF11 mRNA levels in INS-1E beta cells in response to high glucose stimulation INS-1E cells were seeded in six-well plates at a density of $3 \times 10^{5}$ cells per well in the presence of $11.1 \mathrm{mmol} / \mathrm{l}$ glucose. Next day, medium was replaced by medium containing $2.8 \mathrm{mmol} / \mathrm{l}$ glucose. After $24 \mathrm{~h}$, cells were washed with PBS and exposed to medium containing either 2.8 or $25 \mathrm{mmol} / \mathrm{l}$ glucose for 6-48 h. Finally, cells were harvested for analysis of KLF11 mRNA expression levels using qPCR (see below).

$q P C R$ Total RNA was isolated using the RNeasy Mini Kit (Qiagen), treated with Turbo DNA-free Kit (Ambion/Applied Biosystems, Darmstadt, Germany) and checked for contamination with genomic DNA by PCR with primers for rat hypoxanthine phosphoribosyltransferase gene (HPRT) (Quantitect Rn_HPRT1; Qiagen). RNA free from genomic DNA was reverse transcribed with Superscript III and oligo(dT) primers (both Invitrogen). cDNA samples were investigated for mRNA levels by qPCR using Lightcycler 2.0 (Roche) and Faststart DNA Master+ CYBR Green I kit (Roche). Concentrations of mRNA were calculated from standard curves for each specific primer pair (dilution series: 1:1, 1:10, 1:100, 1:1,000 and 1:10,000) by Light Cycler Software 3.5 (Roche). All samples and standards were analysed in triplicates. Primer pairs for rat HPRT (Quantitect Rn_HPRT1), rat Ins1 (Quantitect Rn_INS1), rat Ins2 (Quantitect Rn_INS2) and rat MAOB (Quantitect Rn_MAOB) were obtained from a commercial supplier (Qiagen). Product length of all Quantitect primer pairs is $125 \mathrm{bp}$. See above for primers for $r K L F 11$. qPCR conditions were: $95^{\circ} \mathrm{C}$ for $10 \mathrm{~min} ; 40$ cycles of $95^{\circ} \mathrm{C}$ for $15 \mathrm{~s}, 55^{\circ} \mathrm{C}$ (Quantitect primers) or $64^{\circ} \mathrm{C}$ ( $r K L F 11$ primers) for $10 \mathrm{~s}$ and 
$72^{\circ} \mathrm{C}$ for $20 \mathrm{~s}$; followed by melting curve analysis for specificity of qPCR products.

Glucose-induced insulin secretion from INS-1E beta cells INS$1 \mathrm{E}$ cells were seeded in six-well plates at a density of $3 \times$ $10^{5}$ cells per well in the presence of $11.1 \mathrm{mmol} / 1$ glucose. Next day, medium was replaced by medium containing $2.8 \mathrm{mmol} / \mathrm{l}$ glucose. After $24 \mathrm{~h}$ cells were washed twice with PBS and exposed to serum-free medium containing either 2.8 or $25 \mathrm{mmol} / 1$ glucose. After $1 \mathrm{~h}$, medium samples were collected and centrifuged for $5 \mathrm{~min}$ at $16,000 \mathrm{~g}$. Supernatant fractions were transferred to new tubes and stored at $-20^{\circ} \mathrm{C}$ until measurement of secreted insulin using the High Range Rat Insulin ELISA kit (Mercodia, Uppsala, Sweden).

EMSA One microlitre of in vitro translated reactions was added to $5 \times$ EMSA buffer $\left(100 \mathrm{mmol} / 1 \mathrm{KPO}_{4}, \mathrm{pH} 7.9\right.$, $5 \mathrm{mmol} / \mathrm{l}$ EDTA, $5 \mathrm{mmol} / \mathrm{l}$ dithiothreitol and $20 \%$ glycerol) and additionally supplemented with $50 \mathrm{mmol} / \mathrm{K} \mathrm{KCl}, 1 \mu \mathrm{g}$ poly-dIdC and $40,000 \mathrm{cpm} / \mu \mathrm{l}{ }^{32} \mathrm{P}$-labelled double-stranded oligonucleotide. The following DNA probes were generated by annealing two oligonucleotides, followed by a fill-in reaction with Klenow polymerase and dGTP, dCTP, dTTP and $\alpha-{ }^{32}$ P-labelled dATP: GC box, 5'-GATCAAAGAGCCC CGCCCTGCAGCC-3'; GC box mutant, 5'-GATCAAAGA GCCCCGAACTGCAGCC-3'; CACCC box, 5'-GATCCG ACCCCCCCACCCCAGGCCC-3'; CACCC box mutant, 5'-GATCCGACCCCCCGATTCCAGGCCC-3'. After incubation on ice for $15 \mathrm{~min}$, samples were loaded onto a $5 \%$ polyacrylamide gel and run in $0.5 \times$ Tris-borate-EDTA buffer for $2 \mathrm{~h}$. For supershift assays, $2 \mu \mathrm{l}$ of KLF11 antiserum (see above) were added and incubated on ice for 20 min before samples were loaded onto the gel.

Statistical analysis All results are presented as means \pm SD. Results were analysed by Bonferroni's multiple comparison test of selected groups (Figs. 3d, 5, 6 and 7) and a paired $t$ test (Fig. 8) or ANOVA using GraphPad Prism 4.0.

\section{Results}

KLF11 expression in pancreas, islets and beta cells, and by plasmid RT-PCR demonstrated endogenous KLF11 mRNA expression in whole rat pancreas, human islets of Langerhans and INS-1E beta cells (Fig. 1a). Western blotting detected endogenous KLF11 protein in nuclear extracts of INS-1E and beta-TC 3 beta cells (Fig. 1b). An additional band around $90 \mathrm{kDa}$ represents an artefact of unknown origin occurring with this antibody in some cell lines, i.e. Jurkat (see manufacturer's data sheet). Western blotting further confirmed functional hKLF11 protein production derived from hKLF11-pcDNA3.1+ in whole cell extracts of transiently transfected INS-1E beta cells (Fig. 1c) and in vitro translation (Fig. 1d).

hKLF11 overexpression decreases hInsP activity INS-1E cells were cotransfected with $1.5 \mu \mathrm{g}-881 \mathrm{hInsP}$-pSEAP or promoterless pSEAP2-Basic and increasing quantities $(0-1.5 \mu \mathrm{g})$ of hKLF11-pcDNA3.1+. The amount of totally transfected DNA per well was adjusted to $3 \mu \mathrm{g}$ DNA by addition of pcDNA3.1+. In this experimental setting $h K L F 11$ overexpression dose-dependently reduced $-881 \mathrm{hInsP}$ activity, which is reflected by the magnitude of SEAP in the supernatants, up to $50 \%$ (Fig. 2). Marginal SEAP contents in pSEAP2-Basic transfected controls displayed background levels which remained unregulated by increasing $h K L F 11$ overexpression. The characteristic of $h K L F 11$ overexpression-induced inhibition of $-881 \mathrm{hInsP}$ activity was not altered
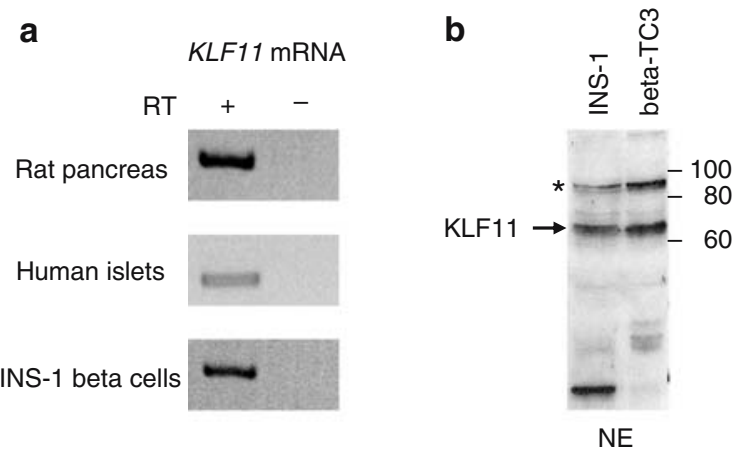

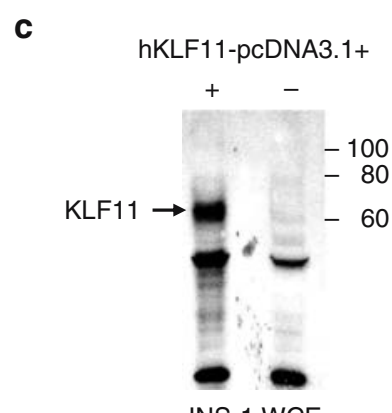

INS-1 WCE

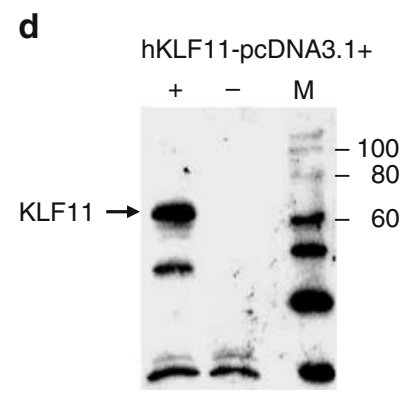

In vitro translation
Fig. 1 KLF11 mRNA and protein expression. a Endogenous KLF11 mRNA is expressed in whole rat pancreas, human islets and INS-1E beta cells. $R T+$, reverse transcriptase added to reaction mix; $R T-$, reverse transcriptase not added. RT- samples serve as a control for contamination of cDNA with genomic DNA. b Endogenous KLF11 protein is produced in INS-1E and beta-TC3 beta cells. Western blots were performed using nuclear extracts $(N E)$. *This band is a known artefact of the antibody used, which occurs in some cell lines, i.e. Jurkat (see manufacturer's data sheet). c INS-1E beta cells transiently transfected with the hKLF11-pcDNA3.1+ expression plasmids demonstrate functional $h K L F 11$ overexpression. Western blots were performed using whole cell extracts $(W C E)$. d Western blotting detected successful in vitro translation of hKLF11 by using hKLF11-pcDNA3.1+ expression plasmids. Displayed are representative results; all experiments, $n=3$ 
a

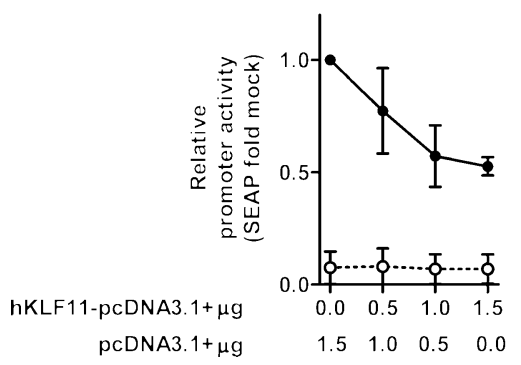

b

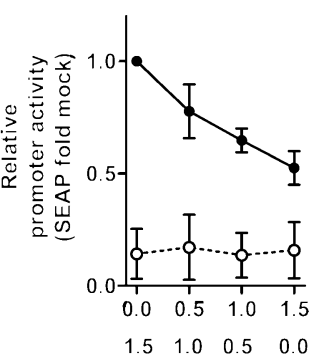

Fig. 2 hKLF11 overexpression-mediated inhibition of cotransfected hInsP. INS-1E beta cells were transiently transfected with different amounts of the hKLF11-pcDNA3.1+ expression plasmids (closed circles) as indicated and cotransfected with either $1.5 \mu \mathrm{g}$ of $-881 \mathrm{hInsP}-\mathrm{SEAP}$ reporter plasmids or $1.5 \mu \mathrm{g}$ of promoterless pSEAP2-Basic empty plasmids as a background control (open circles). Each cotransfection was adjusted with pcDNA3.1+ mock plasmids to a total amount of $3 \mu \mathrm{g}$ transfected DNA. The hKLF11-induced inhibition of hInsP is dose-dependent and stable in standard (a, $11.1 \mathrm{mmol} / \mathrm{l})$ and high (b, $25 \mathrm{mmol} / \mathrm{l})$ glucose conditions. Means $\pm \mathrm{SD} ; n=3$ for $11.1 \mathrm{mmol} / \mathrm{l}$ and $n=5$ for $25 \mathrm{mmol} / 1$ glucose

by high glucose conditions $(25 \mathrm{mmol} / \mathrm{l})$ as compared with standard culture conditions ( $11.1 \mathrm{mmol} / \mathrm{l}$ glucose).

Inhibition of insulin gene expression by KLF11 overexpression, glucose-induced insulin secretion and regulation of endogenous KLF11 mRNA expression by glucose Although a dosedependent KLF11 overexpression-induced increase in $M A O B$ mRNA levels was described in both SH-SY5Y and HepG2 cells [12], we did not observe significant regulation of $M A O B$ gene expression in INS-1E beta cells (not shown). However, qPCR analysis revealed that hKLF11 overexpression significantly reduced Ins 2 gene expression while Ins 1 mRNA levels remained unaffected (Fig. 3a,b). Of note, the observed reduction of about $21 \%$ in the Ins 2 mRNA level is remarkable in the light of approximately onethird transfected cells; $100 \%$ transfection efficiency would result in a $63 \%$ reduction, a value similar to inhibition of hInsP activity by hKLF. The specific inhibition of Ins 2 gene expression is of interest since, in rat beta cells, only the Ins 2 and not the Ins 1 promoter sequence contains a CACCC box element (see below). Functional glucose-induced insulin secretion was tested at the protein level by increasing glucose concentrations in the medium from 2.8 to $25 \mathrm{mmol} / \mathrm{l}$ thereby enhancing 1-h insulin secretion about threefold (Fig. 3c). In contrast to insulin secretion, KLF11 mRNA levels were significantly reduced by high glucose (Fig. 3d).

Binding of KLF11 to the GC and CACCC box Sequence analysis of the hInsP sequence identified the GC box from -340 to -348 and further a CACCC box from -88 to -96 . Positions as well as wild-type and mutated sequences of both boxes are shown in Fig. 4a. EMSA analysis verified binding of in vitro translated hKLF11 to the GC box
(Fig. 4b). Specificity of this binding was demonstrated by antibody-induced supershift. Under the same experimental conditions in vitro translated hKLF11 failed to bind the CACCC box (Fig. 4c).

Inhibition of hInsP 5'-deletion constructs by hKLF11 5'deletion constructs of hInsP were investigated to verify the functional relevance of the GC box for hKLF11-mediated suppression of promoter activity. The position of important binding elements within hInsP and the length of 5 '-deletion constructs are presented in Fig. 5a. In the experimental setting employed, either $0.5 \mu \mathrm{g}$ pcDNA3.1+ (mock control) or $0.5 \mu \mathrm{g}$ hKLF11-pcDNA3.1+ was cotransfected with $0.5 \mu \mathrm{g}$ SEAP reporter plasmids driven by the $-881 \mathrm{hInsP}$ or
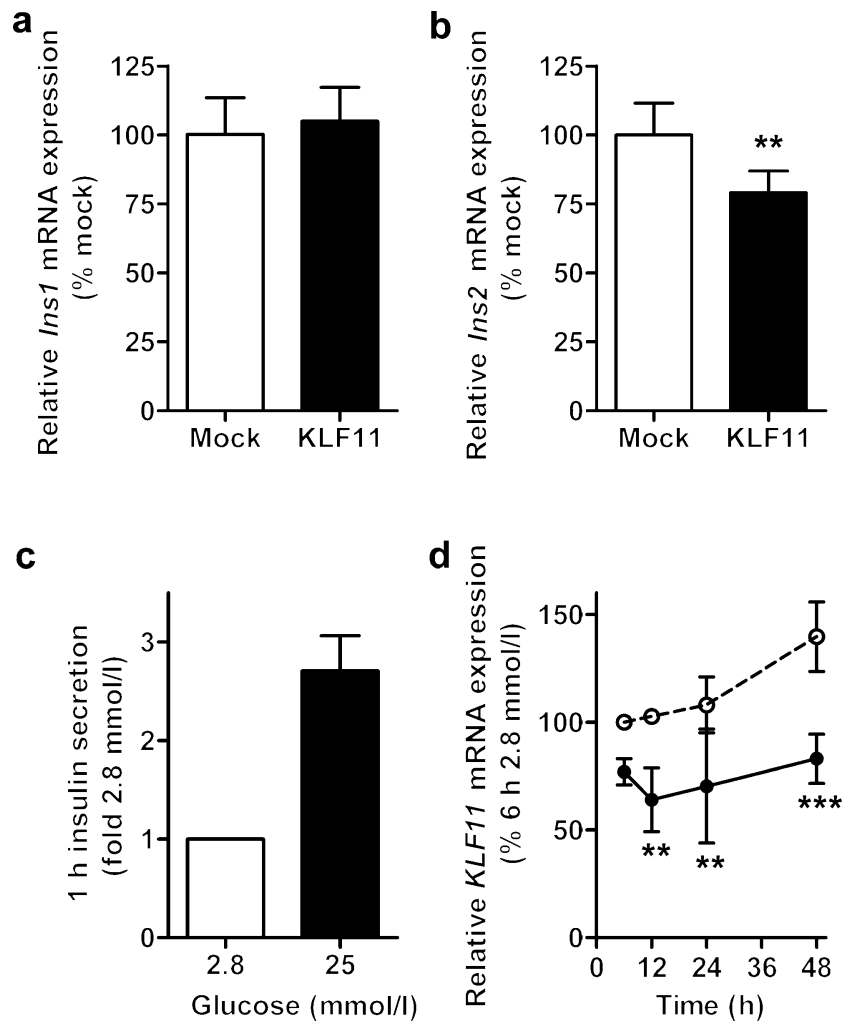

Fig. 3 Inhibition of insulin gene expression by KLF11 overexpression, glucose-induced insulin secretion and regulation of endogenous KLF11 mRNA expression by glucose. a INS-1E beta cells were transfected with rKLF11-pcDNA3.1+ expression plasmids or pcDNA3.1+ empty plasmids (mock control level). After $48 \mathrm{~h}$, cells were harvested for qPCR analysis of Ins $1 \mathrm{mRNA}$ levels. $r K L F 11$ overexpression did not affect Ins 1 gene expression. b In contrast, rKLF11 overexpression significantly reduced Ins 2 gene expression. c INS-1E beta cells were precultured for $24 \mathrm{~h}$ in the presence of $2.8 \mathrm{mmol} / 1$ glucose and then incubated for $1 \mathrm{~h}$ with serum-free medium containing either 2.8 or $25 \mathrm{mmol} / 1$ glucose. High glucose conditions increase insulin secretion nearly threefold, demonstrating glucose responsiveness of the cells investigated. d INS-1E beta cells were precultured for $24 \mathrm{~h}$ in the presence of $2.8 \mathrm{mmol} / 1$ glucose and then exposed to 2.8 (open circles) or $25 \mathrm{mmol} / 1$ (closed circles) glucose. After 6-48 h cells were harvested for qPCR analysis. High glucose conditions significantly reduced endogenous $K L F 11$ mRNA expression. Means \pm SD; all experiments, $n=3 ;{ }^{* *} p<0.01 ; * * * p<0.001$ 
a
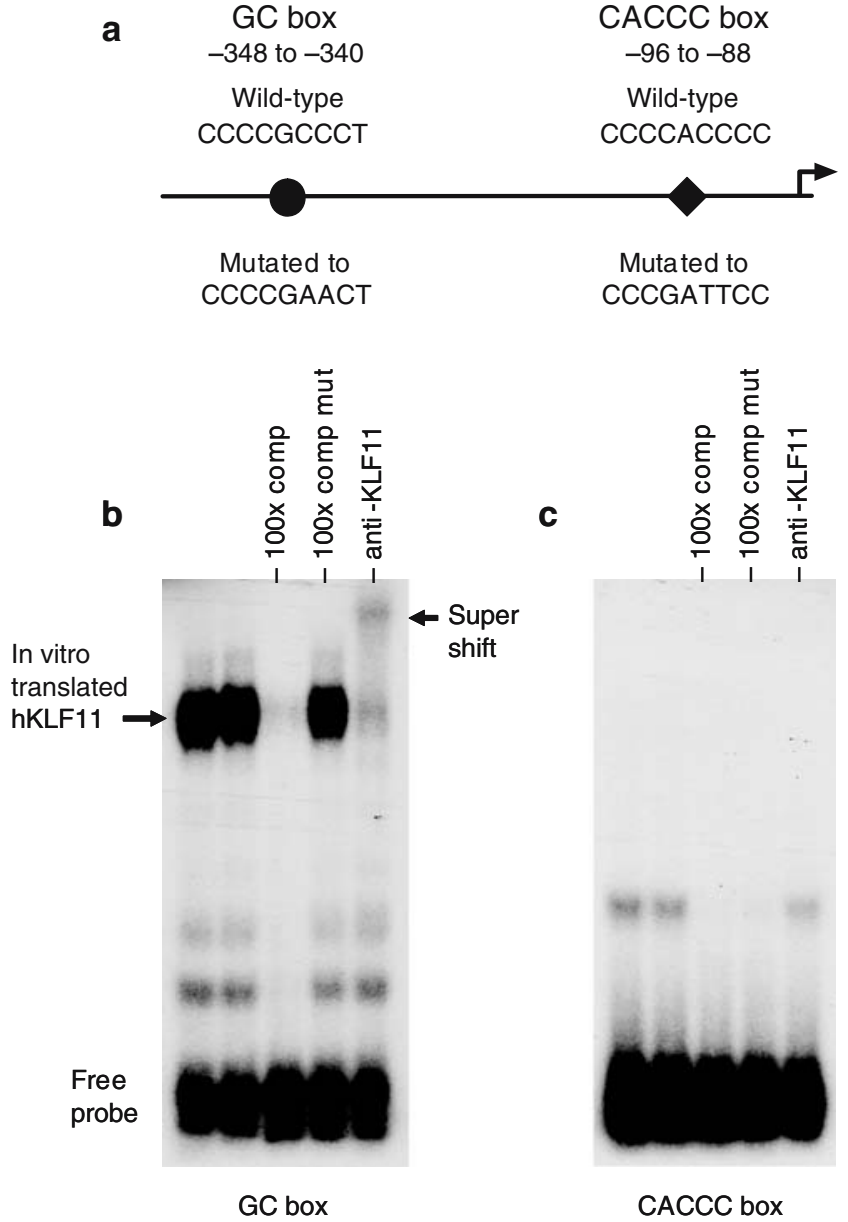

CACCC box mediated inhibition of hInsP depends on DNA sequences upstream of -173 . Although expected, deletion of the GC box did not alter hKLF function. The functional relevance of the CACCC box could not be tested in this setting due to complete loss of KLF11-mediated inhibition of 5'-deletion constructs shorter than -173 .

Effects of GC box and CACCC box mutation on hKLF11inhibited and basal hInsP activity Beta-TC3 beta cells were cotransfected with $-387 \mathrm{hInsP}$-pSEAP (containing wildtype or mutated sequences) and either hKLF-pcDNA3.1+ or pcDNA3.1+ (mock) (Fig. 6). hKLF11 reduced the activity of $-387 \mathrm{hInsP}$-pSEAP to about $40 \%$ of mock control level. In accord with the results from 5 -deletion of hInsP this inhibition was not altered by mutation of the GC box. Although no binding of in vitro translated hKLF11 to the CACCC box was observed in EMSA experiments, CACCC box mutation significantly $(p<0.01)$ reduced hKLF11-

a

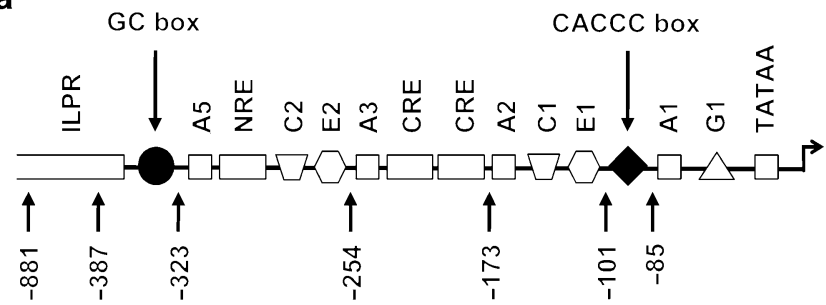

Fig. 4 Interactions of hKLF11 with GC and CACCC boxes. a Position of the GC and CACCC box sequences within hInsP. Wild-type and mutated sequences used in EMSA 25mer probes and reporter plasmids of this study are indicated. b In vitro translated hKLF11 specifically binds to the GC box sequence as demonstrated by a supershift in the presence of an antibody against KLF11. $\mathbf{c}$ In vitro translated hKLF11 did not bind to the CACCC box sequence. Comp, competitor; comp $m u t$, competitor with mutated sequence. All experiments, $n=3$

its 5'-deletion constructs (Fig. 5b). Compared with the initial experiments (dose-dependency of KLF11-mediated inhibition of hInsP) the inhibition by $0.5 \mu \mathrm{g}$ hKLF11pcDNA3.1+ was more pronounced (50\% instead of 30\%). This may be explained by transfection of lower quantities of total DNA ( $1 \mu \mathrm{g}$ instead of $3 \mu \mathrm{g})$, since it is known that high amounts of transfected DNA can alter cellular functions. Besides INS-1E, beta-TC3 beta cells were also investigated. It was confirmed that hKLF11-induced repression of $-881 \mathrm{hInsP}$ activity did not significantly differ between both beta cell lines. In beta-TC3 beta cells, $h K L F 11$ overexpression-induced inhibition of hInsP activity was similar from -881 to -254 (about $60 \%$ inhibition compared with mock control) but was substantially reduced by further deletion to -173 (about $30 \%$ inhibition). Shorter 5 '-deletion constructs were not affected by KLF11 overexpression. These results demonstrate that complete hKLF11-

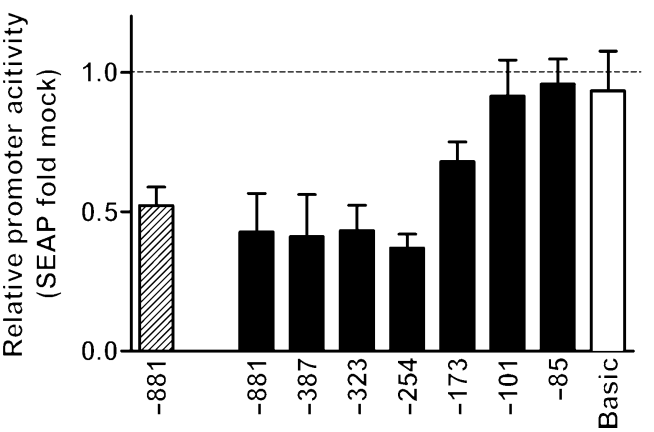

Fig. 5 KLF11-mediated inhibition of 5'-deletion fragments of hInsP. a Schematic delineation of the investigated hInsP full-length fragment and its 5'-deletion constructs subcloned into the promoterless pSEAP2-Basic reporter plasmid. CRE, cAMP response element; $I L P R$, insulin linked polymorphic region; $N R E$, negative regulatory element. b Cotransfection of hInsP-SEAP reporter plasmids with either the hKLF11-pcDNA3.1+ expression plasmid or pcDNA3.1+ empty plasmid (mock control level, dotted line). hKLF11 overexpression-induced inhibition of hInsP activity was similar in INS1E (hatched column) and beta-TC3 beta cells (black columns). Inhibition was stable between -254 to -881 , diminished at -173 and abolished at -101 . Note that inhibition was not altered by deletion of the GC box (-323 and -254). pSEAP2-Basic empty vector (white column) was used as a further control for the absence of non-specific regulation by KLF11. Note that plotted values from hInsP fragments and Basic represent relative changes in promoter activity and not differences in absolute SEAP concentrations. Means \pm SD; $n=5$ 


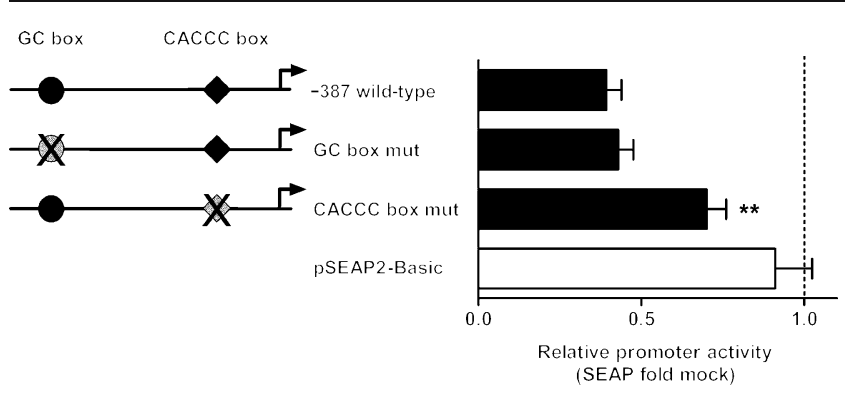

Fig. 6 Functional relevance of GC and CACCC box mutations (box $m u t$ ) for KLF-mediated inhibition of 5'-deletion fragments of hInsP. Beta-TC3 beta cells were cotransfected with -387 hInsP-pSEAP reporter plasmids containing wild-type or mutated (see Fig. 3a) GC and CACCC box sequences and either the hKLF11-pcDNA3.1+ expression plasmid or pcDNA3.1+ empty plasmid (mock control level, dotted line). GC box mutation did not alter hKLF11-induced inhibition of hInsP, while CACCC box mutation significantly diminished this inhibition. pSEAP2-Basic empty vector (white column) was used as a further control for absence of non-specific regulation by KLF11. Note that plotted values from hInsP fragments and Basic represent relative changes in promoter activity and not differences in absolute SEAP concentrations. Means \pm SD; $n=3 ; * * p<0.05$

mediated inhibition of hInsP activity from about $40 \%$ to about $70 \%$ of the mock control level. Interestingly, while calculating relative promoter activities from SEAP data, we observed that absolute SEAP concentrations were substantially lower when the CACCC box was mutated. Therefore, we additionally investigated the functional relevance of the GC box and CACCC box for the basal activity of hInsP by transfection of beta-TC3 beta cells with $-387 \mathrm{hInsP-pSEAP}$ containing wild-type or mutated sequences (Fig. 7). While mutation of the GC box had no significant influence, mutation of the CACCC box rigorously reduced basal hInsP activity to about $25 \%$ of wild-type control levels $(p<0.001)$. We further tested this observation in HEK293 cells (Fig. 8a). The $-323 \mathrm{hInsP}-\mathrm{pSEAP}$ plasmid was used for experiments since its inhibition by $h K L F 11$ overexpression was identical to longer constructs and does not contain the GC box. Even in

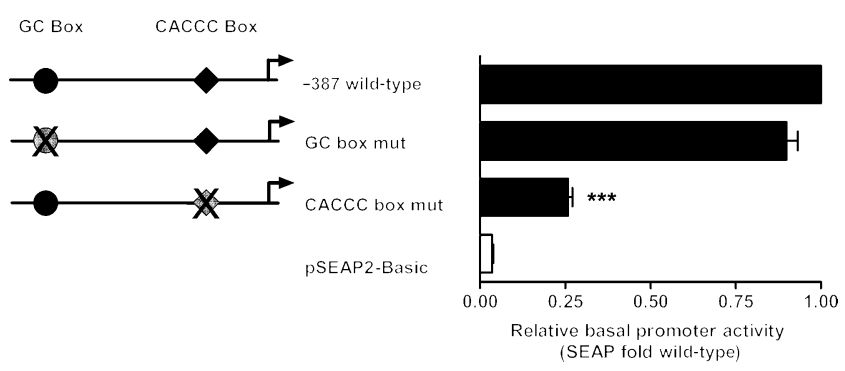

Fig. 7 Functional relevance of GC and CACCC box mutations (box mut) for basal hInsP activity. Beta-TC3 beta cells were transfected with -387 hInsP-pSEAP2-Basic reporter plasmids containing wildtype or mutated (see Fig. 3a) GC and CACCC box sequences or the promoterless pSEAP2-Basic plasmid (background control). Basal activity of hInsP was substantially suppressed by CACCC box mutation, whereas GC box mutation had no effect. Means $\pm \mathrm{SD} ; n=3$; $* * * p<0.001$ a

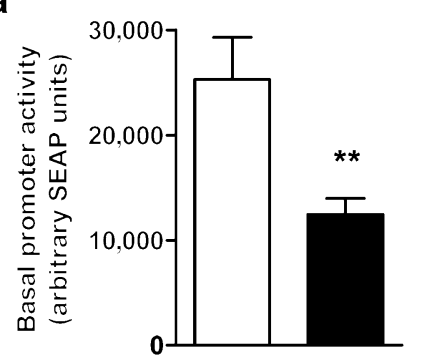

b

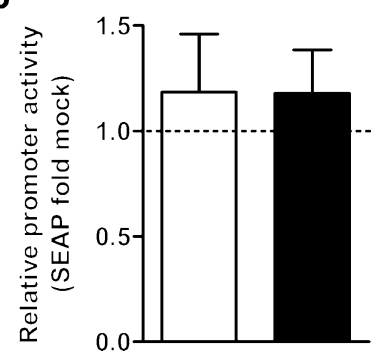

Fig. 8 Functional relevance of CACCC box mutation for basal hInsP activity and KLF11-mediated inhibition of hInsP in HEK293 cells. a Transfection with $-323 \mathrm{hInsP}$-pSEAP reporter plasmids containing wild-type (white column) or mutated (black column) (see Fig. 4a) CACCC box sequences. The $-323 \mathrm{hInsP}$ fragment did not contain a GC box (see Fig. 5a). Also in non-beta cells the CACCC box mutation substantially reduced basal hInsP activity. b Cotransfection with -323hInsP-pSEAP reporter plasmids containing wild-type (white column) or mutated (black column) CACCC box sequences and either hKLF11-pcDNA3.1+ expression plasmids or pcDNA3.1+ empty plasmids (mock control level, dotted line). The hKLF11-induced inhibition of hInsP was completely abolished in non-beta cells. Means \pm SD; all experiments, $n=3 ; * * p<0.01$

HEK293 cells, mutation of the CACCC box significantly reduced basal hInsP activity to about $50 \%$ of wild-type control levels. Finally, compared with mock control level (pcDNA3.1+), hKLF11 did not alter activity of either $-323 \mathrm{hInsP-pSEAP}$ containing wild-type or mutated CACCC box (Fig. 8b).

\section{Discussion}

The TIEG subfamily, consisting of KLF10 and KLF11, is defined by the three N-terminal repressor domains SID/R1, $\mathrm{R} 2$ and R3. These domains have been demonstrated to repress transcription activity $[4,5]$. In this sense, KLF11 acts as a dominant repressor of the caveolin-1 gene [13] and, besides its negative regulation of cell growth in the exocrine pancreas [9], KLF11 overexpression also inhibits cell proliferation in Chinese hamster ovary cells [6]. KLF11 further suppressed oncogene-induced neoplastic transformation in mouse NIH-3T3 cells, and consequently KLF11 mRNA expression was found to be significantly downregulated in a substantial amount (50\%) of investigated pancreatic, breast and kidney tumours [9]. In this context, TGF- $\beta$ inhibits growth of epithelial cells by activation of Smad signalling, which is potentiated through TGF- $\beta$ induced KLF11 by termination of the negative feedback loop imposed by Smad7 [14]. In pancreatic cancer cells with an oncogenic Ras mutation this function is inhibited by ERK/mitogen-activated protein kinase phosphorylation of KLF11, leading to disruption of KLF11-mSin3a interaction, and thereby ends silencing of the Smad7 promoter [14, 15].

In line with these transcription-repressing properties of KLF11, our results demonstrate a dose-dependent inhibition 
of hInsP activity by cotransfected hKLF11 in INS-1E beta cells which is similar in standard and high glucose conditions. The observed hKLF11-mediated inhibition of hInsP activity is underlined by the reduction of Ins 2 gene expression by $r K L F 11$ overexpression. In line with this inhibitory function KLF11 mRNA levels in INS-1E beta cells were repressed by high glucose conditions in which insulin production and secretion are enhanced. Interestingly, in a recent study Neve et al. [10] reported a high glucoseinduced stimulation of KLF11 mRNA expression in INS832/13 beta cells. This opposing observation may be explainable by possible differences between native INS-1E beta cells used in our study and the INS832/13 beta cell line, which is derived from a highly selected INS-1 subclone stably transfected with a plasmid containing the human proinsulin gene [16]. More importantly, Neve et al. [10] demonstrated activation of hInsP by cotransfected FLAG-tagged KLF11 in beta-TC3 beta cells. To be sure that this conflicting finding is not caused by the use of different beta cell lines, we confirmed the hKLF11-induced inhibition of cotransfected hInsP activity observed in INS$1 \mathrm{E}$ beta cells also in beta-TC3 beta cells. Noteworthy, the obtained inhibition was very similar in both beta cell lines demonstrating stable performance of our experimental approach in rodent beta cells independently of the species (rat and mouse). Since the hKLF11-induced inhibition of wild-type and mutated hInsP constructs was completely abolished in HEK293 cells, this function seems to be strictly dependent on the beta cell-specific transcription machinery.

Sp1-like proteins are known to bind with different selectivity to CGCCC or CACCC core sequences in GCrich sites [2]. The requirement of $\mathrm{GC}$ box sequences for functional KLF11-induced repression of promoter activity has been evaluated by several studies $[6,13,14]$. Based on this established concept Neve et al. [10] predicted, although they demonstrate activation and not repression, that KLF11 influenced hInsP via an identified GC box sequence, which they have tested by EMSA. We also verified binding of hKLF11 to the GC box and further investigated the functional relevance of this interaction, but unexpectedly, neither 5'-deletion nor mutation of the GC box altered $h K L F 11$ overexpression-induced inhibition of hInsP activity. This demonstrates that, at least in the context of hInsP, the inhibitory function of hKLF11 is GC box-independent. Moreover, the GC box is also dispensable for the maintenance of basal hInsP activity.

Searching for alternative KLF11-binding sites within hInsP we identified a CACCC box located from -88 to -96. The CACCC sequence was initially reported to be required for KLF11-mediated activation of $\gamma$-globin gene promoter [8], whereas a later study failed to induce significant alterations of $\gamma$-globin gene promoter activity by cotransfected KLF11 [17]. However, KLF11 seems to be expendable for globin gene expression since $K L F 11^{-/}$mice display normal haematopoiesis at all stages of development [18]. Interestingly, $\mathrm{Ou}$ et al. [12] confirmed binding of KLF11 to both Sp1/GC and CACCC sites, thereby influencing the human $M A O B$ promoter as an activator via its $\mathrm{Sp} 1 /$ GC site or as a repressor via its CACCC site. In contrast, we could not detect binding of in vitro translated hKLF11 to the CACCC box sequence within hInsP. This indicates that the observed decrease in hKLF11 inhibitory function due to CACCC box mutation may be caused by indirect interactions. Nevertheless, an involvement of the CACCC box in KLF11 action is supported by the specific KLF11mediated inhibition of Ins 2 gene expression while Ins 1 gene expression remains unaffected. Of note, only the Ins 2 promoter sequence contains a CACCC box.

Interestingly, mutation of the CACCC box substantially downregulated basal activity of hInsP in beta-TC3 beta cells and also HEK 293 cells to about 25 and $50 \%$ of wild-type control levels, respectively. These results suggest that the CACCC box is mainly a target for general transcription factors independently of the beta cell-specific transcription machinery and demonstrates the requirement of a functional CACCC box for the maintenance of normal human proinsulin gene expression. From our results we conclude that this function is independent of the involvement in hKLF-mediated inhibition of hInsP.

Considering all findings we presume that hKLF11 may mainly act via another yet unknown site of hInsP or, more likely, indirectly by interfering with beta cell-specific transcription factors. This reasoning was initiated by the described interaction of KLF1 [19, 20], KLF2 [21], KLF4 $[22,23]$ and $\operatorname{KLF} 13[24,25]$ with the transcriptional cofactors CBP and $\mathrm{p} 300$, which also interact with the pancreatic duodenal homeobox protein (PDX)-1, a major transactivator of proinsulin gene expression in pancreatic beta cells [26, 27]. Of note, KLF13 and KLF11 belong to the same KLF subfamily that is functionally characterised by the SID motif. The speculation of interactions between KLF11 and PDX-1 is supported by the results from 5'-deletion of hInsP demonstrating stepwise decrease of KLF11-induced inhibition. PDX-1 is known to bind to A elements present in the proinsulin promoter region. Although KLF11 function is unaffected by deletion of A5 ( $-254 \mathrm{hInsp}$ fragment), further deletion of A3 (-173hInsp fragment) reduced and deletion of both A3 and A2 (-101hInsP fragment) completely abolished KLF11-mediated inhibition.

In summary, high glucose conditions stimulating insulin production and secretion repress endogenous KLF11 expression in INS-1E beta cells. This is in line with our results characterising hKLF11 as a glucose-independent negative regulator of hInsP in INS-1E and beta-TC3 beta cells. Interestingly, KLF11 specifically reduced Ins 2 but not Ins 1 gene expression in INS-1E beta cells and only the Ins 2 promoter contains a CACCC box. Although we could not 
verify KLF11 interaction with the CACCC box, KLF11mediated inhibition of human proinsulin promoter activity depends on a functional CACCC box, thereby indicating an indirect mechanism. In contrast, and regardless of observed specific binding, the GC box was dispensable for this KLF11 function. Moreover, we demonstrate a new and substantial role for the CACCC box in maintaining basal hInsP activity. In conclusion, these findings may contribute to a better understanding of the complex regulation of proinsulin gene expression and may suggest that dysregulation of KLF11 function and CACCC box mutation have an impact on the development and clinical manifestation of diabetes mellitus.

Acknowledgements This study was supported by the Bundesministerium für Bildung und Forschung (BMBF), Germany (grants FKZ 01GN0114 and FKZ 01GN0115). X. Niu was a recipient of an academic exchange fellowship by the German Academic Exchange Service (DAAD). G. Päth was supported by the Interdisciplinary Center of Clinical Research (IZKF) at the University of Würzburg, Germany (grant Z-4/57 and Z-4/66). C. Limbert is recipient of a fellowship by the European Society of Pediatric Endocrinology (ESPE).

Duality of interest There is no duality of interest to report.

\section{References}

1. Bieker JJ (2001) Kruppel-like factors: three fingers in many pies. J Biol Chem 276:34355-34358

2. Lomberk G, Urrutia R (2005) The family feud: turning off Sp1 by Sp1-like KLF proteins. Biochem J 392:1-11

3. Turner J, Crossley M (1999) Mammalian Kruppel-like transcription factors: more than just a pretty finger. Trends Biochem Sci 24:236-240

4. Zhang JS, Moncrieffe MC, Kaczynski J, Ellenrieder V, Prendergast FG, Urrutia R (2001) A conserved alpha-helical motif mediates the interaction of Sp1-like transcriptional repressors with the corepressor mSin3A. Mol Cell Biol 21:5041-5049

5. Cook T, Gebelein B, Belal M, Mesa K, Urrutia R (1999) Three conserved transcriptional repressor domains are a defining feature of the TIEG subfamily of Sp1-like zinc finger proteins. J Biol Chem 274:29500-29504

6. Cook T, Gebelein B, Mesa K, Mladek A, Urrutia R (1998) Molecular cloning and characterization of TIEG2 reveals a new subfamily of transforming growth factor-beta-inducible Sp1-like zinc finger-encoding genes involved in the regulation of cell growth. J Biol Chem 273:25929-25936

7. Subramaniam M, Harris SA, Oursler MJ, Rasmussen K, Riggs BL, Spelsberg TC (1995) Identification of a novel TGF-beta-regulated gene encoding a putative zinc finger protein in human osteoblasts. Nucleic Acids Res 23:4907-4912

8. Asano H, Li XS, Stamatoyannopoulos G (1999) FKLF, a novel Kruppel-like factor that activates human embryonic and fetal betalike globin genes. Mol Cell Biol 19:3571-3579

9. Fernandez-Zapico ME, Mladek A, Ellenrieder V, Folch-Puy E, Miller L, Urrutia R (2003) An mSin3A interaction domain links the transcriptional activity of KLF11 with its role in growth regulation. EMBO J 22:4748-4758
10. Neve B, Fernandez-Zapico ME, Ashkenazi-Katalan V et al (2005) Role of transcription factor KLF11 and its diabetes-associated gene variants in pancreatic beta cell function. Proc Natl Acad Sci USA 102:4807-4812

11. Schreiber E, Matthias P, Muller MM, Schaffner W (1989) Rapid detection of octamer binding proteins with 'mini-extracts,' prepared from a small number of cells. Nucleic Acids Res 17:6419

12. Ou XM, Chen K, Shih JC (2004) Dual functions of transcription factors, transforming growth factor-beta-inducible early gene (TIEG)2 and Sp3, are mediated by CACCC element and Sp1 sites of human monoamine oxidase (MAO) B gene. J Biol Chem 279:21021-21028

13. Cao S, Fernandez-Zapico ME, Jin D et al (2005) KLF11-mediated repression antagonizes $\mathrm{Sp} 1 /$ sterol-responsive element-binding protein-induced transcriptional activation of caveolin-1 in response to cholesterol signaling. J Biol Chem 280:1901-1910

14. Ellenrieder V, Buck A, Harth A et al (2004) KLF11 mediates a critical mechanism in TGF-beta signaling that is inactivated by ErkMAPK in pancreatic cancer cells. Gastroenterology 127:607-620

15. Ellenrieder V, Zhang JS, Kaczynski J, Urrutia R (2002) Signaling disrupts $\mathrm{mSin} 3 \mathrm{~A}$ binding to the Mad1-like Sin3-interacting domain of TIEG2, an Sp1-like repressor. EMBO J 21:2451-2460

16. Hohmeier HE, Mulder H, Chen G, Henkel-Rieger R, Prentki M, Newgard CB (2000) Isolation of INS-1-derived cell lines with robust ATP-sensitive $\mathrm{K}+$ channel-dependent and -independent glucose-stimulated insulin secretion. Diabetes 49:424-430

17. Zhang P, Basu P, Redmond LC et al (2005) A functional screen for Kruppel-like factors that regulate the human gamma-globin gene through the CACCC promoter element. Blood Cells Mol Diseases 35:227-235

18. Song CZ, Gavriilidis G, Asano H, Stamatoyannopoulos G (2005) Functional study of transcription factor KLF11 by targeted gene inactivation. Blood Cells Mol Diseases 34:53-59

19. Zhang W, Bieker JJ (1998) Acetylation and modulation of erythroid Kruppel-like factor (EKLF) activity by interaction with histone acetyltransferases. Proc Natl Acad Sci USA 95:9855-9860

20. Zhang W, Kadam S, Emerson BM, Bieker JJ (2001) Site-specific acetylation by $\mathrm{p} 300$ or CREB binding protein regulates erythroid Kruppel-like factor transcriptional activity via its interaction with the SWI-SNF complex. Mol Cell Biol 21:2413-2422

21. SenBanerjee S, Lin Z, Atkins GB et al (2004) KLF2 Is a novel transcriptional regulator of endothelial proinflammatory activation. J Exp Med 199:1305-1315

22. Feinberg MW, Cao Z, Wara AK, Lebedeva MA, Senbanerjee S, Jain MK (2005) Kruppel-like factor 4 is a mediator of proinflammatory signaling in macrophages. J Biol Chem 280:3824738258

23. Geiman DE, Ton-That H, Johnson JM, Yang VW (2000) Transactivation and growth suppression by the gut-enriched Kruppellike factor (Kruppel-like factor 4 ) are dependent on acidic amino acid residues and protein-protein interaction. Nucleic Acids Res 28:1106-1113

24. Song CZ, Keller K, Chen Y, Stamatoyannopoulos G (2003) Functional interplay between $\mathrm{CBP}$ and PCAF in acetylation and regulation of transcription factor KLF13 activity. J Mol Biol 329:207-215

25. Song CZ, Keller K, Murata K, Asano H, Stamatoyannopoulos G (2002) Functional interaction between coactivators CBP/p300, PCAF, and transcription factor FKLF2. J Biol Chem 277:70297036

26. Melloul D, Tsur A, Zangen D (2002) Pancreatic duodenal homeobox (PDX-1) in health and disease. J Pediatr Endocrinol Metab 15:1461-1472

27. Ohlsson H, Karlsson K, Edlund T (1993) IPF1, a homeodomaincontaining transactivator of the insulin gene. EMBO J 12:4251-4259 